(C) Springer Verlag. The copyright for this contribution is held by Springer Verlag. The original publication is available at www.springerlink.com. 


\title{
Feature Extraction from Multi-Directional Multi-Resolution Image Transformations for the Classification of Zoom-Endoscopy Images
}

\author{
Roland Kwitt ${ }^{1}$, Andreas Uhl ${ }^{1}$, Michael Häfner ${ }^{2}$ Alfred Gangl $^{2}$, \\ Friedrich $\mathrm{Wrba}^{3}$, Andreas Vécsei ${ }^{4}$ \\ 1 Department of Computer Sciences \\ University of Salzburg \\ A-5020 Salzburg, Austria \\ 2 Department of Gastroenterology and Hepatology \\ Vienna Medical University \\ A-1090 Vienna, Austria \\ 3 Department of Clinical Pathology \\ Vienna Medical University \\ A-1090 Vienna, Austria \\ 4 St. Anna Children's Hospital \\ A-1090 Vienna, Austria
}

Received: date / Revised version: date

\begin{abstract}
In this article, we discuss the discriminative power of a set of image features, extracted from subbands of the Gabor Wavelet Transform and the Dual-Tree Complex Wavelet Transform for the purpose computerassisted zoom-endoscopy image classification. We incorporate color channel information into the classification process and show, that this leads to superior classification results, compared to luminance-channel-only based image analysis.
\end{abstract}

\section{Introduction}

Recent statistics of the American Cancer Society reveal that colorectal cancer is the third most common cancer in men and women and the second leading cause of cancer-related deaths in the $\mathrm{US}^{1}$. Since most colorectal cancers develop from polyps, a regular inspection of the colon is recommended, in order to detect lesions with a malignant potential or early cancer. A common medical procedure to examine the inside of the colon is colonoscopy, which is

1 http://www.cancer.org 
usually carried out with a conventional video-endoscope. A diagnostic benefit can be achieved by employing so called zoom-endoscopes, which achieve a magnification factor of up to 150 by means of an individually adjustable lens. In combination with dye-spraying to enhance the visual appearance (chromo-endoscopy) of the colon mucosa, zoom-endoscopy reveals characteristic surface patterns, which can be interpreted by experienced physicians. Commonly used dyes are either methylene-blue, or indigo-carmine, which both lead to a plastic effect. In the research work of Kudo et al. [14], the macroscopic appearance of colorectal polyps is systematically described and results in the so called pit-pattern classification scheme. In this work, we tackle the problem of computer-assisted pit-pattern classification from the texture-discrimination side and evaluate the discriminative power of features extracted from detail subbands of the Gabor Wavelet Transform and Kingsbury's Dual-Tree Complex Wavelet Transform.

The remainder of this article is structured as follows: in Section 2 we introduce the medical perspective of our work. Section 3 reviews related work on endoscopy image classification. Section 4 presents the proposed feature extraction and classification steps followed by experimental results in Section 5. Section 6 concludes the article with a brief summary of the main points and an outlook on further research.

\section{Pit-Pattern Classification - The Medical Perspective}

Polyps of the colon are a frequent finding and are usually divided into metaplastic, adenomatous and malignant. Since the resection of all polyps is rather time-consuming, it is imperative that those polyps which warrant resection can be distinguished. Furthermore, polypectomy ${ }^{2}$ of metaplastic lesions is unnecessary and removal of invasive cancer may be hazardous. The classification scheme of Kudo et al. divides the mucosal crypt patterns into five groups (pit-patterns I-V, see Fig. 1). While types I and II are characteristic of benign lesions and represent normal colon mucosa or hyperplastic polyps, types III-V represent neoplastic, adenomatous and carcinomatous structures. Table 1 provides a brief overview of the main characteristics of the different pit-patterns. Our classification problem can be stated as follows: the problem to differentiate pit-patterns I and II from III-V will be denoted as the two-class problem, whereas the more detailed discrimination of pit-patterns I-V will be denoted as the six-class problem.

Although at a first glance this classification scheme seems to be straightforward and easy to be applied, it needs some experience and exercising to achieve good results. Correct diagnosis very much relies on the experience of the endoscopist as the interpretation of pit-patterns may be challenging [9]. Additionally, inter-observer variability of magnification chromoendoscopy has been described at least for Barrett's esophagus [17]. This inter-observer variability may to a lesser degree be also present in the interpretation of

\footnotetext{
${ }^{2}$ the process of removing polyps
} 


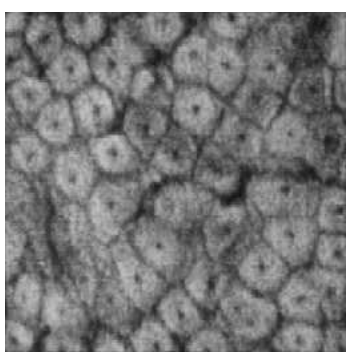

(a) I

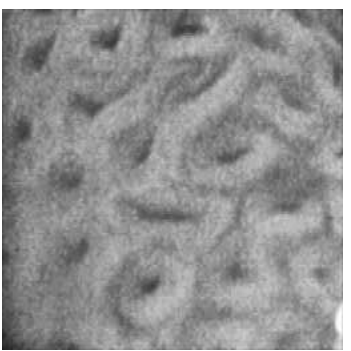

(d) III-S

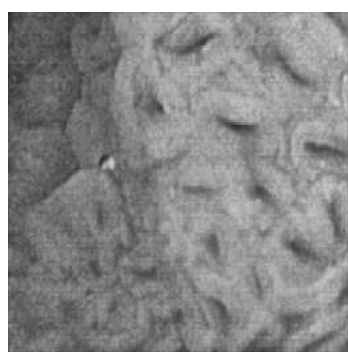

(b) II

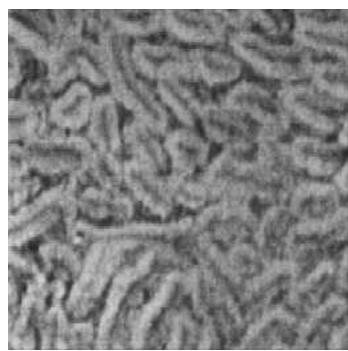

(e) IV

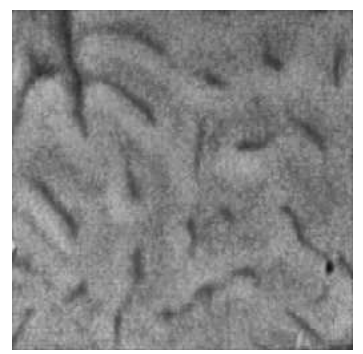

(c) III-L

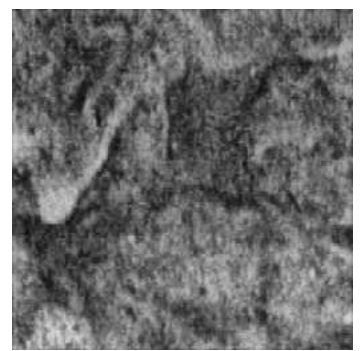

(f) $\mathrm{V}$

Fig. 1 Example images for pit-patterns I-V.

\begin{tabular}{|c|l|}
\hline Pit-Pattern & Characteristics \\
\hline I & roundish pits which designate a normal mucosa \\
II & stellar or papillary pits \\
III-S & small tubular or roundish pits (smaller than type I pits) \\
III-L & large tubular or tubular pits (larger than type I pits) \\
IV & branch-like or gyrus-like pits \\
V & non-structured pits \\
\hline
\end{tabular}

Table 1 Characteristics of the different pit-patterns [15].

pit-patterns of colonic lesions. We therefore want to develop an approach for computer-based pit-pattern classification in order to enhance the quality of diagnostic results.

\section{Related Work}

First, we briefly recapitulate those approaches working on the same image database as we use for our experiments here. In [5] several histogram-based techniques (e.g.: luminance histogram, color-channel histogram) are used to capture the characteristics of the pit-patterns. A k-Nearest Neighbor (k-NN) classifier is then used to classify the images based on histogram intersection used as a distance function. The best classification accuracies - estimated by 
leave-one-out crossvalidation (LOOCV) - in the two- $(85.6 \%)$ and six-class $(67.3 \%)$ problem are achieved using three-dimensional color histograms.

In [6] the authors propose to use wavelet-based texture descriptors for feature extraction. More precisely, the classical 2-D DWT and the 2-D wavelet packet decomposition with local discriminant basis (LDB) [18] are used. In the classification stage, the performance of Support Vector Machines (SVM) and k-NN classification is evaluated on each color-channel specific feature set individually. The best LOOCV accuracy is achieved using features computed on the red color-channel of the RGB model with $75 \%$ in the two-class and $57 \%$ in the six-class case.

A completely different approach is presented in $[3,4]$, where the authors compute a set of texture-descriptors from the outputs of the Discrete Cosine Transform (DCT) and the Discrete Fourier Transform (DFT). Features are either computed from non-overlapping pixel blocks in the DCT domain or from adaptively sized rings in the Fourier domain. Concatenation of the feature vectors of each color-channel is then used to incorporate color-information. The authors employ a Bayes normal classifier [2] together with feature subset selection to classify the endoscopy images. The best reported LOOCV accuracy in the two-class problem is $97.7 \%$ and $86.36 \%$ in the six-class problem.

Another approach that is completely based on grayscale-image analysis is presented in [16], where the marginal distributions of wavelet detail subbands computed from the DT-CWT are modeled by two-parameter Weibull distributions. Maximum-likelihood estimates of the distribution parameters are then composed into feature vectors and are used for k-NN classification. Even though this approach is based on the luminance-channel only, the experiments show superior results when compared to the early approaches in $[5,6]$.

In the work of [10], so called color wavelet covariance features are computed for a set of color models, including RGB and LAB. The image database of this work consists of endoscopic video frames extracted from video sequences, which were acquired during colonoscopy. The approach is based on computing classical cooccurrence matrices from the detail subbands of wavelet-decomposed color-channels. The feature vectors are then composed by a subset of the commonly used Haralick features [7]. Dependencies between color-channels are incorporated by calculating covariances between equal features of different color-channel subbands. However we have to emphasize that the classification problem of [10] is a binary one.

\section{Feature Extraction and Classification}

Each image of our database is decomposed by the Gabor Wavelet Transform (GWT) [1] and Kingsbury's Dual-Tree Complex Wavelet Transform (DT-CWT) [11]. Both transforms aim to remedy two known shortcomings of the maximally decimated 2-D Discrete Wavelet Transform (DWT): the 


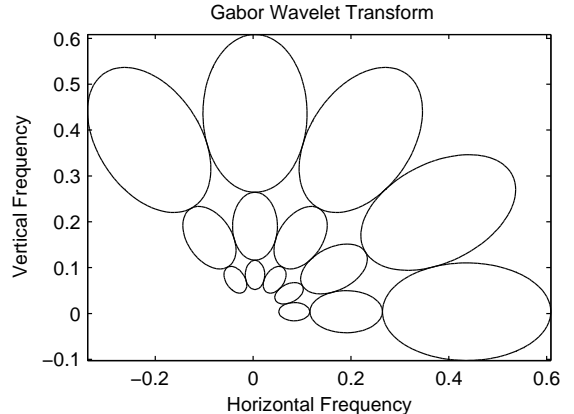

(a) GWT (Half-Peak Magnitude)

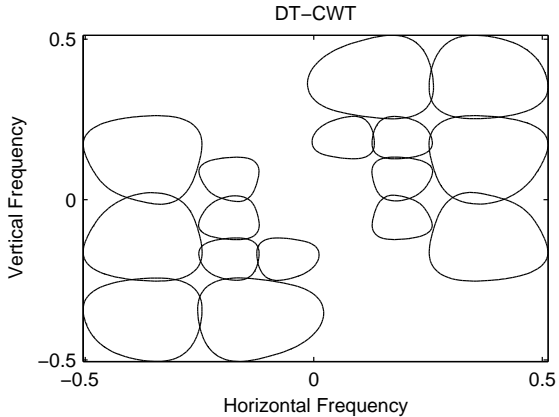

(b) DT-CWT (70\%-Peak Magnitude)

Fig. 2 Exemplary frequency responses of the GWT and the DT-CWT.

inability to capture directional information, which results from the fact that the wavelet filters of the 2-D DWT are separable and real and the lack of shift-invariance caused by downsampling. With respect to our classification problem, it is important to look at the effects of these disadvantages. Just consider the fact that the process of colonoscopy is subject to varying physical conditions. We will thus have to deal with shifted images showing the same type of pit-pattern. Producing different coefficients in that case (shift-dependency) might lead to different image features and as a consequence lead to different classification results. Furthermore, the images exhibit structures directed at orientations other than vertical, horizontal or diagonal $\left( \pm 45^{\circ}\right)$ (see Fig. 1), which cannot be captured by the 2-D DWT.

The GWT is parametrized by the number of orientations, the number of desired scales and the lower- $\left(U_{l}\right)$ and upper $\left(U_{u}\right)$ center frequencies of interest, which are incorporated in the calculation of the scaling factor for the mother wavelet. Since the GWT leads to a non-orthogonal basis, redundancy is minimized by choosing the scaling factor so that the half-peak magnitudes of the filter responses touch each other. An exemplary frequency partitioning of the GWT is shown in Fig. 2(a). The computation of the GWT is usually performed in the Fourier domain where convolutions with the filters reduce to simple multiplications. An inverse Fourier transform then gives the filtered image, which we denote as a subband (adhering to common wavelet terminology). Regarding the concrete parametrization of the GWT, the authors in [1] found that four scales and six orientations, with center-frequencies $\left(U_{l}, U_{u}\right)=(0.05,0.4)$ were optimal for their problem. This led to a final scaling factor of two, where the center-frequencies are one octave apart. In our studies, we adopt the number of orientations and scales, but we vary the upper and lower center frequencies between $0.02 \leq U_{l} \leq$ 0.08 and $0.1 \leq U_{u} \leq 0.4$ to study the effect on the classification results.

The second image transformation we employ here, the DT-CWT, provides an efficient realization of a complex wavelet transform, which is approximately shift-invariant and leads to a fixed number of six detail sub- 
bands, capturing image details oriented at $\approx \pm 15^{\circ}, \pm 45^{\circ}$ and $\pm 75^{\circ}$. In 2-D, the DT-CWT is only four-times redundant and allows an efficient implementation based on four parallel classic 2-D DWTs [19]. The key concept of the DT-CWT is, that the filters in each tree are designed so that the real wavelets they generate form an approximate Hilbert transform pair. Together, they constitute an analytic signal (e.g.: complex wavelet), that is supported on only one-half of the frequency axis $(w>0)$. The $70 \%$ Peak magnitudes of the DT-CWT filter responses are shown in Fig. 2(b). Since the number of orientations in the DT-CWT is fixed to six, we set the decomposition depth to four in order to allow a comparision to the GWT.

Our feature extraction step is based on the assumption, that the distributions of the subband coefficients $\left\{x_{i n}\right\}_{1 \leq n \leq L_{i}}, x_{i n} \in \mathbb{C}$ of subband $i$ characterize the pit-patterns. The total number of coefficients of subband $i$ is denoted by $L_{i}$. Since both the GWT and DT-CWT lead to complex coefficients, we consider the corresponding magnitudes $\left|x_{i n}\right|$. We compute the classic mean $\mu_{i}$ and standard deviation $\sigma_{i}$ of the magnitudes of the transform coefficients, given by

$$
\mu_{i}=\frac{1}{L_{i}} \sum_{j=1}^{L_{i}}\left|x_{i j}\right|, \quad \sigma_{i}=\left(\frac{1}{L_{i}} \sum_{j=1}^{L_{i}}\left(\left|x_{i j}\right|-\mu_{i}\right)^{2}\right)^{\frac{1}{2}}
$$

to constitute a general feature vector $\mathbf{v}=\left[\mu_{1}, \sigma_{1}, \ldots, \mu_{K}, \sigma_{K}\right]^{T}$ for a given image. The same image features have already been successfully used in $[1,8]$ for texture image retrieval. The total number of subbands $K \in \mathbb{N}$ depends on the number of orientations and scales. In our setting of four scales and six orientations we obtain $K=24$ subbands, which results in 48 features per image.

To incorporate color information into the classification process, we first decompose each image into its color channels, transform each channel separately and calculate the image features according to Eq. (1). In our setup here, we will work with RGB images, which leads to three features vectors $\mathbf{v}_{R}, \mathbf{v}_{G}$ and $\mathbf{v}_{B}$ per image. To show, that color channel information can improve classification results, we also compute a feature vector $\mathbf{v}_{L}$ for the luminance (L) channel, which represents the grayscale image. Regarding this setup, it is reasonable to vary the low- and upper- center-frequencies of the GWT, since it is doubtful that the same parameter setting is optimal for all color channels.

Since we either obtain three feature vectors per RGB image or one feature vector for the luminance channel, the question arises, how to combine the color information to obtain the best classification results. A first intuitive approach is to concatenate the feature vectors of the color channels for each image into one big feature vector $\mathbf{v}=\left[\mathbf{v}_{R} \mathbf{v}_{G} \mathbf{v}_{B}\right]^{T}$ with $3 \cdot 48=144$ dimensions. Another approach to tackle this problem is, to use a so called multiclassifier (MC), with three single classifiers operating on the color-channel specific feature sets. The outputs of the classifiers are then combined by 


\begin{tabular}{|c|c|c|c|c|c|}
\hline I & II & III-L & III-S & IV & V \\
\hline 126 & 72 & 62 & 18 & 146 & 60 \\
\hline
\end{tabular}

Table 2 Number of image samples per pit-pattern class (ground truth).

using the combining rules introduced by Kittler et al.[13]. In multi-classifier terminology, the resulting classifier is termed a parallel multi-classifier. We will use k-Nearest Neighbor classifiers [2] as building blocks for our experiments and the euclidean metric as a distance function between the feature vectors. To obtain a reasonable error prediction, we employ the method of leave-one-out cross-validation (LOOCV) [2]. The LOOCV results will be given by the final classification accuracy, which is defined as the number of correctly classified samples divided by the total sample size.

\section{Experimental Results}

In this section, we present the experimental results of our work. Our image database consists of 484 images acquired in 2005/2006 at the Department of Gastroenterology and Hepatology (Medical University of Vienna) using a zoom-endoscope (Olympus Evis Exera CF-Q160ZI/L) with a magnification factor of 150 . To enhance visual appearance, dye-spraying with indigo-carmine was applied and biopsies or mucosal resections were taken to obtain a histopathological diagnosis. For pit-patterns I,II and V, biopsies were taken, since these types need not be removed. Lesions of pit-patterns III-S/III-L and IV have been removed endoscopically. Table 2 lists the number of image samples per class.

Before actually transforming the images, we perform two image-quality enhancing pre-processing steps: first, we use contrast-limited adaptive histogram equalization (CLAHE) [20] with $8 \times 8$ tiles and an uniform distribution for constructing the contrast transfer function. Second, we apply a Gaussian blur with $\sigma=0.5$ using a $3 \times 3$ mask. Regarding the choice of filters for the DT-CWT, we use Kingsbury's Q-Shift $(14,14)$-tap filters (for decomposition levels $\geq 2$ ) in combination with (13,19)-tap near-orthogonal filters (for decomposition level 1) [12]. The technical reasons for using different filter sets are explained in [19]. In order to exemplify the advantages of the multidirectional transformations, we further decompose each image by the critically sampled 2-D DWT using a four-scale decomposition with Daubechies 6 -tap filters and compute image features according to Eq. (1). The 2-D DWT leads to three detail subbands at each decomposition level, hence we obtain 24-dimensional feature vectors per color channel. The LOOCV results for the red, green and blue color channel are given in Table 3. We can see, that in all tests the features obtained from the red color channel lead to the best LOOCV results for the two- and six-class problem, which is consistent with the results of [6]. Regarding the parameter $k$ of the k-NN classifier, we ran several tests with $1 \leq k \leq 20$, with $k=1$ leading to the 


\begin{tabular}{|c|c|c|c|c|c|c|c|}
\hline \multirow{2}{*}{ Problem } & \multicolumn{6}{|c|}{ Pit-Patterns } & \multirow{2}{*}{ Total } \\
\hline & I & II & III-L & III-S & IV & $\mathrm{V}$ & \\
\hline \multicolumn{8}{|c|}{ Gabor Wavelet Transform (GWT) } \\
\hline 2-cls., red & \multicolumn{2}{|c|}{85.35} & \multicolumn{4}{|c|}{89.86} & 88.02 \\
\hline 2-cls., blue & \multicolumn{2}{|c|}{84.34} & \multicolumn{4}{|c|}{88.46} & 85.54 \\
\hline 2-cls., green & \multicolumn{2}{|c|}{85.35} & \multicolumn{4}{|c|}{87.06} & 85.54 \\
\hline 6-cls., red & 73.81 & 61.11 & 59.68 & 38.89 & 69.18 & 63.33 & 66.12 \\
\hline 6-cls., blue & 68.25 & 52.78 & 69.36 & 44.44 & 67.81 & 61.67 & 64.26 \\
\hline 6-cls., green & 67.46 & 54.17 & 66.13 & 50.00 & 69.18 & 50.00 & 63.02 \\
\hline \multicolumn{8}{|c|}{ Dual-Tree Complex Wavelet Transform (DT-CWT) } \\
\hline 2-cls., red & \multicolumn{2}{|c|}{90.91} & \multicolumn{4}{|c|}{95.11} & 93.39 \\
\hline 2-cls., blue & \multicolumn{2}{|c|}{84.85} & \multicolumn{4}{|c|}{87.76} & 86.57 \\
\hline 2-cls., green & \multicolumn{2}{|c|}{87.37} & \multicolumn{4}{|c|}{89.51} & 88.64 \\
\hline 6-cls., red & 80.95 & 73.61 & 70.97 & 72.22 & 79.45 & 73.33 & 76.86 \\
\hline 6-cls., blue & 75.40 & 62.50 & 69.36 & 44.44 & 70.55 & 71.67 & 69.63 \\
\hline 6-cls., green & 66.67 & 62.50 & 61.29 & 55.56 & 65.07 & 63.33 & 64.05 \\
\hline \multicolumn{8}{|c|}{ Discrete Wavelet Transform (DWT) } \\
\hline 2-cls., red & \multicolumn{2}{|c|}{71.72} & \multicolumn{4}{|c|}{86.36} & 80.37 \\
\hline 2-cls., blue & \multicolumn{2}{|c|}{72.73} & \multicolumn{4}{|c|}{83.57} & 79.13 \\
\hline 2-cls., green & \multicolumn{2}{|c|}{64.65} & \multicolumn{4}{|c|}{74.38} & 74.38 \\
\hline 6-cls., red & 65.87 & 41.67 & 58.06 & 27.78 & 63.01 & 58.33 & 58.06 \\
\hline 6-cls., blue & 59.52 & 54.17 & 45.16 & 33.33 & 63.01 & 38.33 & 54.34 \\
\hline 6-cls., green & 58.73 & 38.89 & 50.00 & 11.11 & 56.16 & 38.33 & 49.59 \\
\hline
\end{tabular}

Table 3 Comparison of the two- and six-class LOOCV results for both multidirectional transforms and the classic 2-D DWT, using a 1-NN classifier. The best results for each color channel are marked bold.

\begin{tabular}{|c|c|c|c|c||c|c|c|c|}
\hline & \multicolumn{4}{|c||}{ 2-class } & \multicolumn{4}{c|}{ 6-class } \\
\hline & Red & Blue & Green & Gray & Red & Blue & Green & Gray \\
\hline$U_{l}$ & 0.06 & 0.04 & 0.08 & 0.08 & 0.06 & 0.06 & 0.06 & 0.04 \\
\hline$U_{u}$ & 0.40 & 0.20 & 0.30 & 0.40 & 0.40 & 0.30 & 0.20 & 0.10 \\
\hline$\rightarrow a$ & 1.88 & 1.71 & 1.55 & 1.71 & 1.88 & 1.71 & 1.49 & 1.36 \\
\hline
\end{tabular}

Table 4 Optimal parameter settings for the Gabor Wavelet Transform.

best results in almost all cases. All further results were obtained adopting this setting. Table 4 lists the best parameter settings $\left(U_{l}, U_{u}\right)$ for the GWT, together with the resulting scale factor $a$. We point out, that the optimal parameters for the color channels are in no case equal to the parameters $\left(U_{l}, U_{u}\right)=(0.05,0.4)$ in [1] and additionally differ for the two classification problems. This justifies our approach to vary the center frequencies.

Next, we compare the results of the combining schemes to the luminancechannel-only results. Regarding the parallel multi-classifier, we used the product, mean, median, maximum, minimum and majority voting [13] rule 


\begin{tabular}{|c|c|c|c|c|c|c|c|}
\hline \multirow{2}{*}{ Problem } & \multicolumn{6}{|c|}{ Pit-Patterns } & \multirow{2}{*}{ Total } \\
\hline & I & II & III-L & III-S & IV & $\mathrm{V}$ & \\
\hline \multicolumn{8}{|c|}{ Gabor Wavelet Transform (GWT) } \\
\hline 2-cls., gray & \multicolumn{2}{|c|}{88.39} & \multicolumn{4}{|c|}{88.12} & 88.22 \\
\hline 2-cls., concat. & \multicolumn{2}{|c|}{89.90} & \multicolumn{4}{|c|}{93.36} & 91.94 \\
\hline 2-cls., MC & \multicolumn{2}{|c|}{90.91} & \multicolumn{4}{|c|}{93.01} & 92.15 \\
\hline 6-cls., gray & 66.67 & 65.28 & 62.90 & 50.00 & 67.12 & 66.67 & 65.50 \\
\hline 6-cls., concat. & 81.74 & 68.06 & 75.81 & 55.56 & 73.97 & 68.33 & 73.97 \\
\hline 6-cls., MC & 83.33 & 56.94 & 75.81 & 38.89 & 83.56 & 61.67 & 74.17 \\
\hline \multicolumn{8}{|c|}{ Dual-Tree Complex Wavelet Transform (DT-CWT) } \\
\hline 2-cls., gray & \multicolumn{2}{|c|}{87.88} & \multicolumn{4}{|c|}{91.61} & 90.08 \\
\hline 2-cls., concat. & \multicolumn{2}{|c|}{92.93} & \multicolumn{4}{|c|}{98.60} & 96.28 \\
\hline 2-cls., MC & \multicolumn{2}{|c|}{93.94} & \multicolumn{4}{|c|}{97.20} & 95.87 \\
\hline 6-cls., gray & 73.02 & 68.06 & 66.13 & 55.56 & 69.86 & 70.00 & 69.42 \\
\hline 6-cls., concat. & 85.71 & 69.44 & 88.71 & 83.33 & 82.88 & 78.33 & 81.81 \\
\hline 6-cls., MC & 84.92 & 66.67 & 82.26 & 55.56 & 84.93 & 75.00 & 79.55 \\
\hline \multicolumn{8}{|c|}{ Discrete Wavelet Transform (DWT) } \\
\hline 2-cls., gray & \multicolumn{2}{|c|}{73.23} & \multicolumn{4}{|c|}{77.27} & 75.62 \\
\hline 2-cls., concat. & \multirow{2}{*}{\multicolumn{2}{|c|}{$\begin{array}{l}87.88 \\
79.80\end{array}$}} & \multicolumn{4}{|c|}{93.36} & 91.12 \\
\hline 2-cls., MC & & & \multicolumn{4}{|c|}{91.96} & 86.98 \\
\hline 6-cls., gray & 64.29 & 47.22 & 54.84 & 33.33 & 55.48 & 41.67 & 53.93 \\
\hline 6-cls., concat. & 78.57 & 58.33 & 77.42 & 33.33 & 72.60 & 60.00 & 69.63 \\
\hline 6-cls., MC & 79.37 & 52.78 & 70.97 & 22.22 & 71.92 & 60.00 & 67.56 \\
\hline
\end{tabular}

Table 5 Comparison of the LOOCV results for the luminance channel and the color information combining schemes. The MC results were obtained by using the product rule. The top results for both classification problems are marked bold.

for combining the 1-NN classifier outputs. In case of equal votes in majority voting, ties are broken in favor of the larger class. The LOOCV accuracies are listed in Table 5. For all three transformations, the product rule performed best for the two- and six-class problem using the multi-classifier. The overall best LOOCV results were obtained by using the DT-CWT features together with feature vector concatenation. However, due to the very marginal differences in LOOCV accuracies, especially regarding the concatenation and MC approach, it is hard to provide a general answer which combining scheme to prefer. At least, we can conclude that both combining schemes are superior to the luminance channel features, which lead to the worst performance.

When comparing the differences between the three image transformations for a fixed combining scheme, we see that the DT-CWT features show the best performance, especially for the complicated six-class problem. It is further interesting that by relying on the DT-CWT features of the red color channel alone (see Table 3), we achieve higher LOOCV rates than by using the DWT or GWT features, no matter which combining scheme we 
choose. This leads to the conclusion, that the DT-CWT is the best choice in our experimental setting, since it is computationally less expensive and less redundant than the GWT and clearly outperforms both the DWT and GWT in terms of LOOCV accuracy.

\section{Conclusion}

In this article presented an approach for computer-assisted pit-pattern classification. By employing two multi-directional, multi-resolution image transformations, we have shown that the properties of directional-selectivity and approximate shift-invariance improve classification accuracy. Further, our results indicate that grayscale-image analysis is inferior to color-image analysis in our problem, no matter which combining scheme we choose. In addition to that we could show that the simple concatenation scheme for combining feature vectors from different color channels leads to very good results when compared to the more advanced classifier combining rules. Further work on this topic will include a study on the impact of other color models, since we conducted our experiments on RGB images only. Switching to other color-spaces like LAB or HSV might improve classification results. Furthermore, we plan to study the impact of classifiers other than k-NN to allow more complex decision boundaries.

Acknowledgements This work is funded by the Austrian Science Fund (FWF) under Project No. L366-N15.

\section{References}

1. W.Y. Ma B.S. Manjunath. Texture features for browsing and retrieval of image data. IEEE Transactions on Pattern Analysis and Machine Intelligence, 18(8):837-842, August 1996.

2. K. Fukunaga. Introduction to Statistical Pattern Recognition. Morgan Kaufmann, 2nd edition, 1990.

3. M. Häfner, L. Brunauer, H. Payer, R. Resch, F. Wrba, A. Gangl, A. Vécsei, and A. Uhl. Pit pattern classification of zoom-endoscopic colon images using DCT and FFT. In P. Kokol, V. Podgorelec, D. Micetic-Turk, M. Zorman, and M. Verlic, editors, Proceedings of the IEEE International Symposium on Computer-Based Medical Systems (CBMS'07), pages 159-164, Maribor, Slovenia, June 2007. IEEE Computer Society CPS.

4. M. Häfner, L. Brunauer, H. Payer, R. Resch, F. Wrba, A. Gangl, A. Vécsei, and A. Uhl. Pit pattern classification of zoom-endoscopical colon images using evolved Fourier feature vectors. In K. Diamantaras, T. Adali, I. Pitas, J. Larsen, T. Papadimitriou, and S. Douglas, editors, Proceedings of the 2007 IEEE Machine Learning for Signal Processing Workshop (MLSP'07), pages 99-104, Thessaloniki, Greece, August 2007. IEEE.

5. M. Häfner, Ch. Kendlbacher, W. Mann, W. Taferl, F. Wrba, A. Gangl, A. Vécsei, and A. Uhl. Pit pattern classification of zoom-endoscopic colon 
images using histogram techniques. In Johannes R. Sveinsson, editor, Proceedings of the 7th Nordic Signal Processing Symposium (NORSIG 2006), pages 58-61, Reykavik, Iceland, June 2006. IEEE.

6. M. Häfner, M. Liedlgruber, F. Wrba, A. Gangl, A. Vécsei, and A. Uhl. Pit pattern classification of zoom-endoscopic colon images using wavelet texture features. In W. Sandham, D. Hamilton, and C. James, editors, Proceedings of the International Conference on Advances in Medical Signal and Image Processing (MEDSIP'06), Glasgow, Scotland, UK, July 2006. paper no. 0038.

7. R. M. Haralick, Dinstein, and K. Shanmugam. Textural features for image classification. IEEE Transactions on Systems, Man, and Cybernetics, 3:610621, November 1973.

8. S. Hatipoglu, Sanjit K. Mitra, and Nick G. Kingsbury. Texture classification using dual-tree complex wavelet transform. In Seventh International Conference on Image Processing and Its Applications, volume 1, pages 344-347, Manchester, UK, July 1999.

9. D.P. Hurlstone. High-resolution magnification chromoendoscopy: Common problems encountered in "pit pattern" interpretation and correct classification of flat colorectal lesions. American Journal of Gastroenterology, 97:1069-1070, 2002.

10. Stavros A. Karkanis. Computer-aided tumor detection in endoscopic video using color wavelet features. IEEE Transactions on Information Technology in Biomedicine, 7(3):141-152, September 2003.

11. Nick G. Kingsbury. The dual-tree complex wavelet transform: a new technique for shift invariance and directional filters. In Proceedings of the IEEE Digital Signal Processing Workshop, DSP '98, pages 9-12, Bryce Canyon, USA, August 1998.

12. Nick G. Kingsbury. Complex wavelets for shift invariant analysis and filtering of signals. Applied and Computational Harmonic Analysis, 10(3):234-253, May 2001.

13. J. Kittler, M. Hatef, R.P.W. Duin, and J. Matas. On combining classifiers. IEEE Transactions on Pattern Analysis and Machine Intelligence, 20(3):226239, March 1998.

14. S. Kudo, S. Hirota, T. Nakajima, S. Hosobe, H. Kusaka, T. Kobayashi, M. Himori, and A. Yagyuu. Colorectal tumorous and pit pattern. Journal of Clinical Pathology, 47:880-885, 1994.

15. S. Kudo, S. Tamura, T. Nakajima, H. Yamano, H. Kusaka, and H. Watanabe. Diagnosis of colorectal tumorous lesions by magnifying endoscopy. Gastrointestinal Endoscopy, 44(1):8-14, July 1996.

16. R. Kwitt and A. Uhl. Modeling the marginal distributions of complex wavelet coefficient magnitudes for the classification of zoom-endoscopy images. In Proceedings of the IEEE Computer Society Workshop on Mathematical Methods in Biomedical Image Analysis (MMBIA'07), pages 1-8, Rio de Janeiro, Brasil, 2007.

17. A. Meining, T. Rösch, R. Kiesslich, M. Muders, F. Sax, and W. Heldwein. Inter- and intra-observer variability of magnification chromoendoscopy for detecting specialized intestinal metaplasis at the gastroesophageal junction. Endoscopy, 36(2):160-164, February 2004.

18. N. Saito and R.R. Coifman. Local discriminant bases. In A.F. Laine and M.A. Unser, editors, Wavelet Applications in Signal and Image Processing II, volume 2303 of SPIE Proceedings, pages 2-14, San Diego, CA, July 1994. 
19. I. W. Selesnick, R. G. Baraniuk, and N. Kingsbury. The dual-tree complex wavelet transform - a coherent framework for multiscale signal and image processing. IEEE Signal Processing Magazine, 22(6):123-151, November 2005.

20. K. Zuiderveld. Contrast limited adaptive histogram equalization. In Paul S.

Heckbert, editor, Graphics Gems IV, pages 474-485. Morgan Kaufmann, 1994. 